\title{
METODOLOGIA PARA PRESERVAÇÃO DO FUNGICIDA MANCOZEBE EM AMOSTRAS DE SOLO
}

\author{
Ana Claudia Pires da Rosa e Mônica Regina da Costa Marques* \\ Instituto de Química, Universidade do Estado do Rio de Janeiro, Rua São Francisco Xavier, 524, 20550-900 Rio de Janeiro - RJ, Brasil \\ Daniel Vidal Pérez \\ Empresa de Pesquisa Agropecuária do Rio de Janeiro - EMBRAPA, Rua Jardim Botânico, 1024, 22460-000 Rio de Janeiro - RJ, Brasil
}

Recebido em 9/11/10; aceito em 4/4/11; publicado na web em 10/6/11

\begin{abstract}
METHOD FOR PRESERVATION OF THE FUNGICIDE MANCOZEB IN SOIL SAMPLES. The influence of temperature, storage time and the addition of hydrochloride L-cysteine in the preservation of the fungicide mancozeb was studied under field conditions in the surface layer of a Cambisol collected in an area planted with cabbage in Nova Friburgo - RJ, Brazil. The results of this study showed an efficiency of up to $42 \%$ in the preservation of the fungicide in the samples treated with cysteine and stored at low temperatures compared to those not treated and kept at room temperature.
\end{abstract}

Keywords: soil; pesticide; dithiocarbate.

\section{INTRODUÇÃO}

O sistema de produção agrícola com cultivo de grandes áreas exige a utilização intensiva de insumos agrícolas. Dentre eles, os pesticidas estão entre os mais importantes para a diminuição dos altos índices de perda na produção. Por outro lado, o uso inadequado dessas substâncias pode acarretar sérias consequências para o homem e o meio ambiente. ${ }^{1}$ Os sinais de impacto nem sempre são imediatos, podendo ser crônicos, quando interferem na expectativa de vida, crescimento, fisiologia, comportamento e reprodução dos organismos, ou ecológicos, quando interferem na disponibilidade de alimentos, de habitats e na biodiversidade. ${ }^{2}$ Por causa destes efeitos nocivos, tornam-se imprescindíveis o acompanhamento e a quantificação dos pesticidas na água, no solo, no ar e nos alimentos para preservar a saúde do homem e do meio ambiente. ${ }^{1}$

Quando uma molécula de pesticida alcança o solo, ela pode seguir diferentes rotas: ser absorvida pelas plantas, transformada, retida no solo ou ainda transportada para diferentes compartimentos ambientais. ${ }^{3}$ A transformação de um pesticida no solo consiste na alteração de sua estrutura molecular por meios bióticos, isto é, por ação do metabolismo dos micro-organismos no solo; ou abióticos, onde a molécula é transformada pela ação de componentes físicos ou químicos do ambiente. A hidrólise e a fotólise são as principais reações de degradação abiótica de pesticidas no solo. A reação de hidrólise pode ser influenciada pelo $\mathrm{pH}$ do meio, pela temperatura e pela sorção do pesticida. ${ }^{3}$

Os ditiocarbamatos (DTC), que pertencem ao grupo dos fungicidas, são compostos orgânicos sulfurados derivados do ácido bisditiocarbâmico. A maioria dos DTC foi desenvolvida no início da década de 30, entretanto ainda são largamente empregados tanto sozinhos quanto combinados com outros fungicidas. ${ }^{4}$

Os etileno-bis-ditiocarbamatos (EBDC), subgrupo mais importante dos DTC, são amplamente empregados na agricultura como fungicidas não sistêmicos, controlando grande espectro de fungos. Sua aplicação mundial extensiva pode ser atribuída a sua baixa toxicidade aguda e à curta permanência no ambiente. Todos os EBDC possuem

*e-mail: monicamarques@uerj.br um esqueleto orgânico comum $\left(\mathrm{C}_{4} \mathrm{H}_{6} \mathrm{~N}_{2} \mathrm{~S}_{4}\right)$ diferindo apenas do íon metálico presente na molécula. Os principais EBDC comerciais são: manebe (EBDC-Mn), mancozebe (Figura 1, EBDC-Mn-Zn) e zinebe (EBDC-Zn). Além das estruturas químicas semelhantes, todos os EBDC podem gerar dissulfeto de carbono, ser metabolizados a etilenotioureia e quelar íons metálicos, como o cobre. ${ }^{5}$

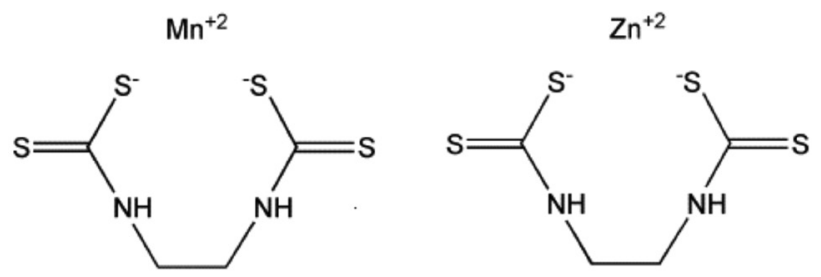

Figura 1. Fórmula estrutural do mancozebe (etileno bis-ditiocarbamato de manganês e zinco)

A detecção dos ditiocarbamatos foi estudada por vários pesquisadores. Alguns deles demonstraram que a cromatografia líquida de alta eficiência (CLAE) permite a determinação de DTC, entretanto a cromatografia gasosa de alta resolução (CGAR) e a espectrofotometria na região do ultravioleta visível (UV-VIS) apresentam melhores sensibilidades para a determinação de traços. ${ }^{6}$

A detecção de DTC em hortaliças e frutas por espectroscopia UV-VIS é muito utilizada pelos órgãos públicos de fiscalização, por ser de baixo custo. Este método se baseia na determinação do $\mathrm{CS}_{2}$ gerado após a digestão ácida dos DTCs, na presença de cloreto estanoso. ${ }^{7} \mathrm{O}$ limite de quantificação (LQ) de resíduos de DTC em hortaliças por este método é de $0,40 \mathrm{mg} \mathrm{kg}^{-1} \mathrm{CS}_{2}$ e o limite de detecção (LD), de $0,28 \mathrm{mg} \mathrm{kg}^{-1}$. Modificações deste método têm sido relatadas principalmente em relação ao tipo de matriz ambiental e ao pré-tratamento da amostra, com o objetivo de reduzir o tempo e as complicações desse método..$^{5,8}$

Segundo Prestes et al. ${ }^{9}$ na determinação de resíduos de pesticidas, são necessárias etapas prévias de preparo da amostra, devido a vários fatores: baixas concentrações do analito; propriedades químicas distintas e complexidade das matrizes. Além disso, como nem sempre é possível 
realizar análise imediatamente após a amostragem, a etapa de preservação torna-se de extrema importância. Para a maioria dos pesticidas, a preservação a $-20{ }^{\circ} \mathrm{C}$ já é suficiente. Entretanto, alguns pesticidas podem ser hidrolisados e oxidados facilmente. ${ }^{10}$ No caso de pesticidas sulfurados, como o EDBC, a oxidação deste grupo gera etilenotioureia (ETU). ${ }^{11}$

Kobayashi et al. ${ }^{12}$ relataram que agentes redutores como ácido ascórbico e cloridrato de L-cisteína em amostras vegetais são capazes de retardar a degradação do mancozebe impedindo a oxidação de EBDC a etilenotioureia (ETU). Os autores observaram uma degradação de $10 \%$ quando uma solução mancozebe foi submetida a $50{ }^{\circ} \mathrm{C}$ sob vácuo. Em condições de refluxo, a conversão foi de $81 \%$. No entanto, quando as soluções foram tratadas com cloridrato de L-cisteína e sujeitas às mesmas condições, não se observou a formação de ETU.

Considerando a importância da preservação do analito para sua detecção e da rápida degradação do mancozebe em solos, ${ }^{13}$ o presente estudo teve como objetivos avaliar a capacidade redutiva do cloridrato de L-cisteína, a temperatura e o tempo de estocagem na preservação do fungicida mancozebe em amostras de solo provenientes de uma lavoura de couve.

\section{PARTE EXPERIMENTAL}

\section{Descrição da área}

O estudo foi conduzido em condições de campo no solo de uma região entre São Lourenço e Salinas no distrito de Campo de Coelho (coordenadas geográficas: 22²1'03.91" S e 4240'19.86" W). A região está localizada na área de entorno do Parque Estadual dos Três Picos pertencente ao Município de Nova Friburgo, região serrana do Estado de Rio de Janeiro denominada "Cinturão Verde", que é responsável por $70 \%$ da produção interna de verduras, legumes e frutas. Nesta região o solo predominante é o cambissolo. ${ }^{14}$

\section{Caracterização do solo}

Amostras do solo antes da aplicação do pesticida foram coletadas na profundidade de $0-10 \mathrm{~cm} .{ }^{13}$ Durante a coleta, as amostras foram acondicionadas em sacos plásticos limpos e lacradas para posterior caracterização. As características físicas e químicas da amostra do solo foram realizadas nos laboratórios da Embrapa/Solos (RJ), segundo o manual de análises de solos. ${ }^{13}$

\section{Aplicação do mancozebe em uma cultura de couve da família das Brassicaceae}

Para este estudo, foi utilizado o fungicida mancozebe - ingrediente ativo formulado pela Empresa - Du Pont do Brasil S.A., comercializado como Manzate ${ }^{\circledR} 800$.

Aplicaram-se $5 \mathrm{~L}$ de solução do fungicida em dose recomendada pelo fabricante para agricultura convencional, utilizando-se pulverizador costal cobrindo uma área equivalente a $36 \mathrm{~m}^{2}$. A aplicação do mancozebe na área experimental foi realizada 30 dias após o plantio de uma cultura de couve (Brassica oleracea L.). A amostragem foi conduzida após $24 \mathrm{~h}$ da aplicação do fungicida na profundidade de até $10 \mathrm{~cm}$ do solo, com o auxílio de um trado. Para o transporte do campo até o laboratório, $1 \mathrm{~kg}$ da amostra de solo foi acondicionada em sacola plástica, lacrada e colocada em caixa de isopor com gelo.

\section{Ensaios de preservação de amostras de solo com mancozebe}

A amostra foi dividida em duas porções iguais. Na primeira, foram borrifados $75 \mathrm{~mL}$ de solução saturada de cloridrato de L-cisteína mono-hidratada P.A. ${ }^{12}$ e na outra, o mesmo volume de água deioniza- da. As amostras foram homogeneizadas manualmente e novamente divididas em duas partes, resultando em 4 subamostras que foram armazenadas sob as condições descritas na Tabela 1 .

Tabela 1. Condições de armazenagem das subamostras

\begin{tabular}{lcc}
\hline Subamostra & Adição de cloridrato de L-cisteína & T armazenamento \\
\hline 1 & Não & $\pm 25^{\circ} \mathrm{C}$ \\
2 & Não & $-20{ }^{\circ} \mathrm{C}$ \\
3 & Sim $^{\mathrm{a}}$ & $\pm 25^{\circ} \mathrm{C}$ \\
4 & Sim $^{\mathrm{a}}$ & $-20^{\circ} \mathrm{C}$ \\
\hline
\end{tabular}

a: amostra foi borrifada com $75 \mathrm{~cm}^{3}$ de solução saturada de cloridrato de L-cisteína

\section{Determinação do mancozebe no solo}

A determinação do mancozebe foi realizada segundo o procedimento de utilizado por Arcanjo ${ }^{5}$ para solos. 12,50 g da subamostra de solo e $175 \mathrm{~mL}$ da solução ácida digestora foram adicionados em um balão de fundo redondo de capacidade $500 \mathrm{~mL}$ acoplado a um condensador de refluxo (Figura 2). Mediante aquecimento a $105^{\circ} \mathrm{Ce}$ fluxo de nitrogênio (180 a $200 \mathrm{~mL} \mathrm{~min}^{-1}$ ), o CS 2 liberado foi deslocado para o recipiente 2, localizado abaixo do condensador (ampliação da Figura 2), contendo $15 \mathrm{~mL}$ do reagente complexante de cobre (II).

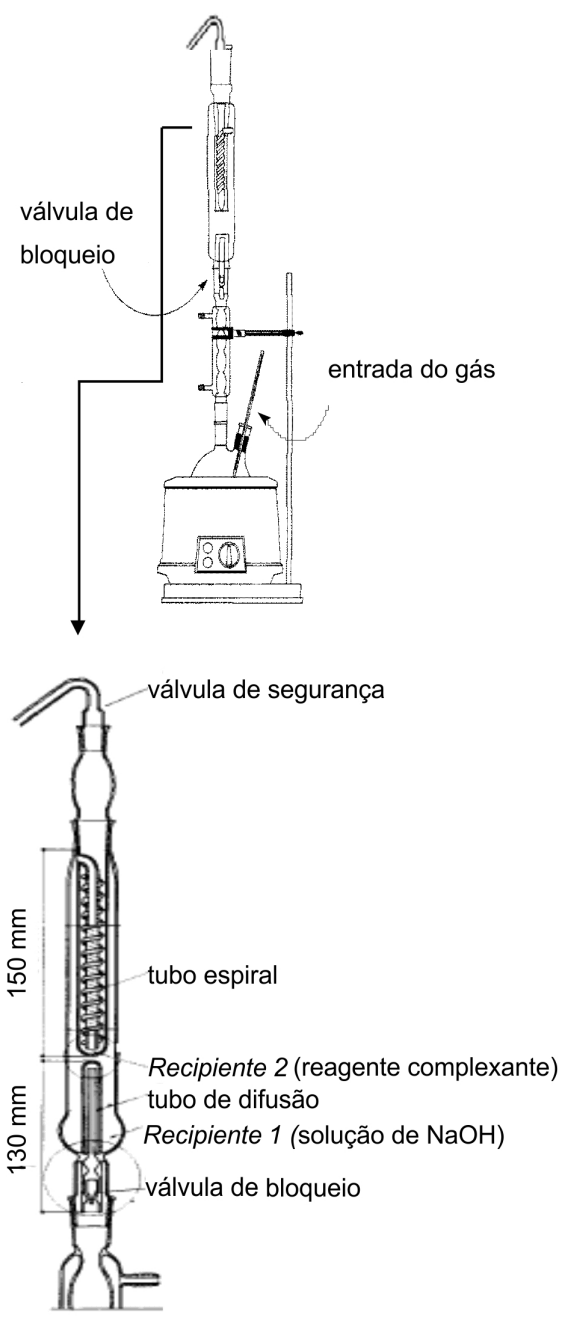

Figura 2. Aparelhagem para a determinação de ditiocarbamatos 
A absorvância do complexo amarelo (dietilditiocarbamato de cobre II) foi medida em espectrofotômetro UV-VIS (Femto modelo $600 \mathrm{~s})$ num comprimento de onda de $435 \mathrm{~nm}$. Um conjunto de soluções de diferentes concentrações de $\mathrm{CS}_{2}(0,1000-1,000 \mu \mathrm{g} \mathrm{mL})$ foi preparado a partir de solução estoque (diluição de $1 \mathrm{~mL}$ de $\mathrm{CS}_{2}$ grau espectroscópico em balão volumétrico de $50 \mathrm{~mL}$ com etanol) para a construção de uma curva de calibração, por meio da qual foi determinada a quantidade de $\mathrm{CS}_{2}$ gerado. $\mathrm{O}$ teor de mancozebe final é proporcional à quantidade gerada de $\mathrm{CS}_{2}$. Cada amostra foi analisada em triplicata.

\section{Solução ácida digestora}

A $500 \mathrm{~mL}$ de $\mathrm{HCl}$ P.A., adicionaram-se 31,25 g de cloreto estanoso P.A. Completou-se o volume até $2,5 \mathrm{~L}$ com água deionizada.

\section{Reagente complexante de cobre}

Em balão volumétrico de $1 \mathrm{~L}$, adicionou-se $0,048 \mathrm{~g}$ de acetato de cobre (II) mono-hidratado P.A., $100 \mathrm{~g}$ de dietanolamina P.A. e completou-se o volume com etanol.

\section{Tratamento estatístico dos dados}

A análise de variância para o estudo de preservação das amostras foi realizada segundo o delineamento experimental inteiramente casualizado, com os tratamentos distribuídos em arranjo fatorial $2 \mathrm{x}$ $2 \times 3$ (duas temperaturas, dois tratamentos com e sem cisteína e três diferentes tempos de análise), com três repetições. Para a análise dos dados, foram utilizados os softwares estatísticos: SAS System for Windows (Statistical Analysis System) ${ }^{15}$ e SISVAR. ${ }^{16}$ As médias foram comparadas pelo teste de Scott-Knott ${ }^{17}$ a $5 \%$ de probabilidade.

\section{RESULTADOS E DISCUSSÃO}

As propriedades granulométricas do solo coletado na profundidade de 0-10 $\mathrm{cm}$ demonstraram tratar de um solo de textura franco $\operatorname{arenosa}^{14}$ (Tabela 2). Em solos deste tipo, a lixiviação de pesticidas no solo é facilitada e torna vulnerável a contaminação das águas subterrâneas. ${ }^{14}$

Tabela 2. Análise textural do solo estudado

\begin{tabular}{cccc}
\hline Areia $(\%)$ & Silte $(\%)$ & Argila $(\%)$ & Silte/argila (\%) \\
\hline 63,0 & 18,6 & 18,4 & 1,01 \\
\hline
\end{tabular}

A concentração de $\mathrm{H}^{+}$no complexo de troca foi alta $\left(8 \mathrm{cmol}_{\mathrm{c}} / \mathrm{kg}\right.$ - Tabela 3). Este valor de acidez é típico de solo de regiões tropicais que são intensamente intemperizadas. ${ }^{13}$

O solo foi considerado distrófico, pois apresentou percentual de saturação das bases (V) menor que 50\% (Tabela 3), característico de solos ácidos. ${ }^{14} \mathrm{O}$ teor de carbono no solo é médio $\left(24,5 \mathrm{~g} \mathrm{~kg}^{-1}\right)$, segundo a Embrapa. ${ }^{13} \mathrm{O}$ elevado teor de fósforo $\left(203 \mathrm{mg} \mathrm{kg}^{-1}\right)$, não comum neste tipo de solo, está relacionado ao histórico de plantio de batata na região, décadas atrás.

\section{Preservação da amostra}

$\mathrm{Na}$ amostra controle, não foi detectada a presença de $\mathrm{CS}_{2}$ pelo método descrito neste trabalho, indicando ausência de resíduo de mancozebe no solo estudado. A ausência de $\mathrm{CS}_{2}$ na amostra controle fortificada com cloridrato de L-cisteína indica que a digestão do cloridrato de L-cisteína não produz $\mathrm{CS}_{2}$.

A amostra estudada foi coletada $24 \mathrm{~h}$ após aplicação do fungicida no solo e analisada no mesmo dia. A concentração inicial detectada foi de aproximadamente $5,0 \mathrm{mg} \mathrm{kg}^{-1}$. O restante da amostra foi dividido em 4 subamostras (Tabela 1).

O método estatístico de Snott-Knott ${ }^{17}$ foi utilizado para classificar as médias obtidas nos experimentos (Tabela 4) e separá-las em grupos (adição ou não de cisteína, tempo e temperatura de armazenamento). Este método de classificação de médias atribui letras para diferenciar uma média de outra. Médias em uma mesma coluna que possuem a mesma letra minúscula indicam que as concentrações obtidas não diferem estatisticamente. Assim, a concentração obtida para a amostra sem cisteína e a armazenada a $25^{\circ} \mathrm{C}$ por 15 dias $(1,29$ - denominação bB) é estaticamente similar à análise após 35 dias $(0,63 \mathrm{bA})$, enquanto que, na mesma linha, os valores contendo as mesmas letras maiúsculas indicam que as médias obtidas são consideradas equivalentes (não possuem diferença significativa). Quando isso acontece, se considera que a diferença entre os valores das médias equivalentes é devida à variação ao acaso.

Tabela 4. Teste de médias Scott-Knott nos parâmetros analisados

Concentrações média obtidas de $\mathrm{CS}_{2}\left(\mathrm{mg} \mathrm{kg}^{-1}\right)$ Adição de cisteína

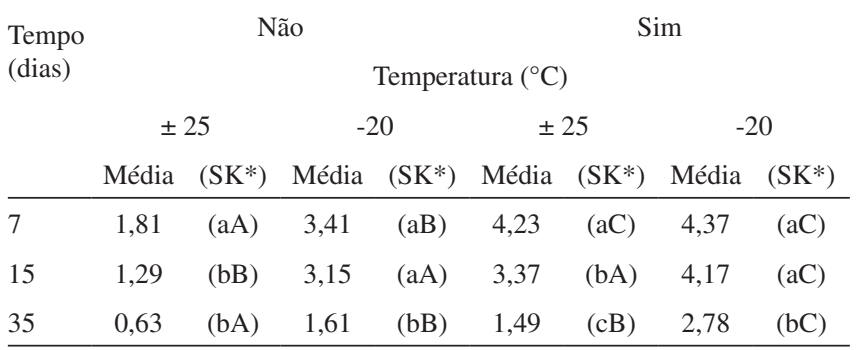

*Scott-Knott a $5 \%$ de probabilidade. Na mesma coluna: médias com letras minúsculas iguais não diferem estatisticamente; na mesma linha: médias contendo mesmas letras maiúsculas são similares estatisticamente; concentração inicial: 4,96 de $\mathrm{CS}_{2} \mathrm{mg} \mathrm{kg}^{-1}$.

As amostras tratadas com cloridrato de L-cisteína apresentaram concentrações de $\mathrm{CS}_{2}$ superiores às amostras sem cisteína. Para essas amostras, estocadas durante 7 dias, a análise de variância das médias das concentrações de $\mathrm{CS}_{2}$ indicou que tanto sob temperatura de -20 ${ }^{\circ} \mathrm{C}\left(4,37 \mathrm{mg} \mathrm{kg}^{-1}\right)$ como à temperatura ambiente $\left(4,23 \mathrm{mg} \mathrm{kg}^{-1}\right)$ os resultados não apresentaram diferenças estatísticas. Para as amostras mantidas a $-20^{\circ} \mathrm{C}$, os resultados foram semelhantes para 7 e 15 dias de estocagem: 4,37 e 4,17 $\mathrm{mg} \mathrm{kg}^{-1}$, respectivamente. Com isso, para amostras mantidas nessa temperatura, as análises podem ser condu-

Tabela 3. Caracterização físico-química do solo estudado

\begin{tabular}{lcccccc}
\hline $\mathrm{pH}$ & $\mathrm{H}^{+}\left(\mathrm{cmol}_{\mathrm{c}} / \mathrm{kg}\right)$ & $\mathrm{S}\left(\mathrm{cmol}_{\mathrm{c}} / \mathrm{kg}\right)$ & $\mathrm{CTC}\left(\mathrm{cmol}_{\mathrm{c}} / \mathrm{kg}\right)$ & $\mathrm{V}(\%)$ & $\mathrm{Corg}(\%)$ & $\mathrm{P}\left(\mathrm{mg} \mathrm{kg}^{-1}\right)$ \\
\hline 5,6 & 8,0 & 5,5 & 13,6 & 40 & 2,45 & 2,8 \\
\hline
\end{tabular}

$\mathrm{S}$ (bases trocáveis): $\mathrm{Ca}^{2+}+\mathrm{Mg}^{2+}+\mathrm{K}^{+}+\mathrm{Na}^{+}$; CTC: capacidade de troca catiônica; V: índice de saturação por base e calculado pela fórmula 100 x $\mathrm{S} / \mathrm{CTC}$; Corg: carbono orgânico 
zidas com até 15 dias de armazenagem. Entretanto, após 7 dias de estocagem, sem a adição de cisteína e nas temperaturas de -20 e $25^{\circ} \mathrm{C}$, os resultados foram inferiores: $3,41 \mathrm{e} 1,81 \mathrm{mg} \mathrm{kg}^{-1}$, respectivamente.

Em geral, o tratamento com cloridrato de L-cisteína aliado ao armazenamento a baixas temperaturas forneceu resultados superiores, alcançando até $42 \%$ de diferença em relação às amostras não tratadas com cisteína.

\section{CONCLUSÃO}

Os resultados apresentados mostraram que o tratamento das amostras de solo com cloridrato de L-cisteína e armazenamento a $-20{ }^{\circ} \mathrm{C}$ reduziu consideravelmente as perdas do mancozebe antes da análise, por isso, concluiu-se tratar de alternativa eficaz de preservação de amostras de solo com esse fungicida.

\section{REFERÊNCIAS}

1. Moura, L. H. A.; Boaventura, G. R.; Pinelli, M. P.; Quim. Nova 2010, 33, 97; Silva, F. C.; Cardeal, Z. L.; Carvalho, C. R.; Quim. Nova 1999, $22,197$.

2. Luna, A. J.; Sales, L. T.; Silva, R. F.; Agrotóxicos: Responsabilidade de Todos (Uma abordagem da questão dentro do paradigma do desenvolvimento sustentável). Disponível em http://www. segurancaetrabalho.com.br/download/agrotoxicos-responsabilidade.doc. acessada em Maio 2011.

3. Teixeira, S. C. G.; Canela, M. C.; Quim. Nova 2007, 30, 1830; Hirata, R.; Kortzaru, B.; Narciso, E. S.; Arq. Inst. Biol. 2003, 70, 359.
4. Bastos, L. H. P.; Góes, H. C. A.; Cardosos, M. H. W. M.; Gouvea, A. V.; Dias, D. P.; Almeida, R. R. R.; Nóbrega, A.; Abrantes, S.; Quim. Nova 2007, 30, 32 .

5. Arcanjo, M. E.; Dissertação de Mestrado, Universidade do Estado do Rio de Janeiro, Brasil, 2007.

6. Coldwell, M. R.; Pengelly, I.; Rimmer, D. A.; J. Chromatogr., A 2003, 984, 81; Stertz, S. C.; Freitas, R. J.; Pesticidas: R. Ecotox. e Meio Ambiente 2003, 13, 45.

7. Caldas, E. D.; Miranda, M. C. C.; Conceição, M. H.; Souza, L. C. K. R.; Food Chem. Toxicol. 2004, 42, 1883.

8. Cullen, E. T.; Niagara Chemical Division, FMC Corp, Middleport, 1964, vol. 36, 221; Keppel, G. E.; J. AOAC 1971, 54, 528; Vuik, J.; van Dinter, R.; De Vos, R. H.; J. Agric. Food. Chem. 1992, 40, 604.

9. Prestes, O. D.; Friggi, C. A.; Adaime, M. B.; Zanella, R.; Quim. Nova 2009, 32, 1620.

10. Egli, H.; J. Agric. Food. Chem. 1982, 30, 861.

11. Nash, R. G.; Beall, M. L. Jr.; J. Agric. Food. Chem. 1980, 28, 322.

12. Kobayashi, H.; Nishida, M.; Matano, O.; Goto, S.; J. Agric. Food Chem. 1992, 40, 76.

13. Empresa Brasileira de Pesquisa Agropecuária; Manual de métodos de análises de solo, $2^{\mathrm{a}}$ ed., CNPS: Rio de Janeiro, 1997.

14. Lemos R. C.; Santos, R. D.; Manual de descrição e coleta de solo no campo, $2^{\mathrm{a}}$ ed., Embrapa - Centro Nacional de Pesquisa de Solo: Rio de Janeiro, 1984.

15. SAS SYSTEM; The SAS System for Windows Release 8.02; University de Massey, USA, 1999.

16. Ferreira, D. F.; SISVAR; Sisvar versão 4.6 (Build 63); Universidade Federal de Lavras, Brasil, 1999.

17. Scott, A.; Knott, M.; Biometrics 1974, 30, 507. 